\title{
Vécu de la paternité et implication dans les soins périnataux : rencontres avec des hommes africains émigrés en Belgique
}

\section{Dimitri Cauchie, Marielle Bruyninckx et Ornella Cappalonga}

Volume 5, numéro 1, 2015

Engagement paternel et migration

URI : https://id.erudit.org/iderudit/1077306ar

DOI : https://doi.org/10.7202/1077306ar

Aller au sommaire du numéro

Éditeur(s)

Alterstice

ISSN

1923-919X (numérique)

Découvrir la revue

Citer cet article

Cauchie, D., Bruyninckx, M. \& Cappalonga, O. (2015). Vécu de la paternité et implication dans les soins périnataux : rencontres avec des hommes africains émigrés en Belgique. Alterstice, 5(1), 35-44. https://doi.org/10.7202/1077306ar
Résumé de l'article

Notre article présente les résultats d'une recherche menée auprès d'hommes africains, originaires de la région des Grands Lacs et immigrés en Belgique, quant à leur conception et à leur vécu de la paternité et à leur participation au suivi pré- et post-natal. La migration étant susceptible de les avoir menés à concevoir leur paternité dans un nouveau cadre de références culturelles, nous nous sommes interrogés sur les changements potentiels dans la répartition des rôles parentaux et au vécu de la paternité. Nous avons rencontré les sujets de notre échantillon deux fois, vers le huitième mois de grossesse et environ deux semaines après la naissance, afin de mener des entretiens semi-directifs et de faire passer le Questionnaire d'auto-évaluation de la compétence éducative parentale. Les résultats obtenus semblent montrer que ces pères immigrés s'investissent dans les soins périnataux de leur enfant avec attention et motivation. S'ils reconnaissent avoir dû adapter leurs pratiques parentales au contexte sociétal dans lequel ils évoluent (engagement professionnel des femmes, absence de famille élargie...), ils se déclarent satisfaits de remplir leur rôle de père et semblent également envisager leur implication dans une perspective davantage proactive que réactive, n'excluant pas de l'intensifier (par la prise d'un congé parental notamment). Les résultats sont présentés en détail après une brève synthèse de la littérature quant aux évolutions contemporaines des rôles parentaux dans les sociétés occidentales et aux conceptions générales de la paternité dans les sociétés africaines.
C Dimitri Cauchie, Marielle Bruyninckx et Ornella Cappalonga, 2015

Ce document est protégé par la loi sur le droit d'auteur. L’utilisation des services d'Érudit (y compris la reproduction) est assujettie à sa politique d'utilisation que vous pouvez consulter en ligne.

https://apropos.erudit.org/fr/usagers/politique-dutilisation/ 


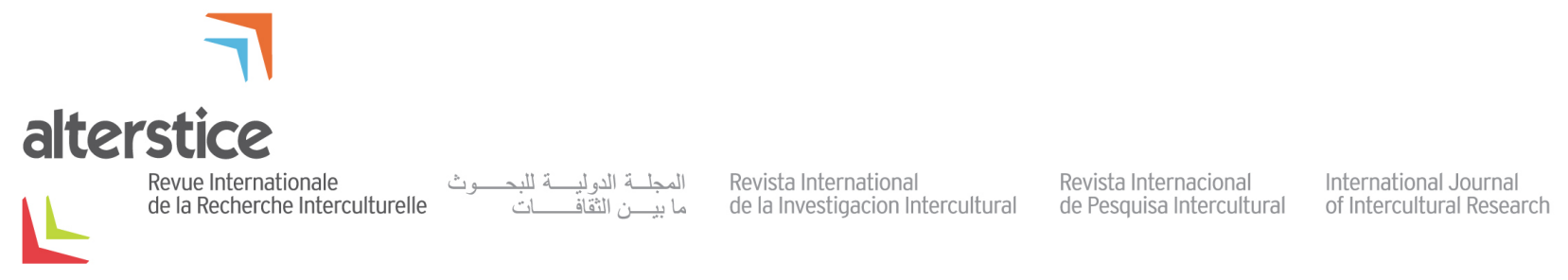

ARTICLE THÉMATIQUE

\title{
Vécu de la paternité et implication dans les soins périnataux : rencontres avec des hommes africains émigrés en Belgique
}

\author{
Dimitri Cauchie ${ }^{1}$, Marielle Bruyninckx ${ }^{1}$ et Ornella Cappalonga ${ }^{1}$
}

\section{Résumé}

Notre article présente les résultats d'une recherche menée auprès d’hommes africains, originaires de la région des Grands Lacs et immigrés en Belgique, quant à leur conception et à leur vécu de la paternité et à leur participation au suivi pré- et post-natal. La migration étant susceptible de les avoir menés à concevoir leur paternité dans un nouveau cadre de références culturelles, nous nous sommes interrogés sur les changements potentiels dans la répartition des rôles parentaux et au vécu de la paternité. Nous avons rencontré les sujets de notre échantillon deux fois, vers le huitième mois de grossesse et environ deux semaines après la naissance, afin de mener des entretiens semi-directifs et de faire passer le Questionnaire d'auto-évaluation de la compétence éducative parentale. Les résultats obtenus semblent montrer que ces pères immigrés s'investissent dans les soins périnataux de leur enfant avec attention et motivation. S'ils reconnaissent avoir dû adapter leurs pratiques parentales au contexte sociétal dans lequel ils évoluent (engagement professionnel des femmes, absence de famille élargie...), ils se déclarent satisfaits de remplir leur rôle de père et semblent également envisager leur implication dans une perspective davantage proactive que réactive, n'excluant pas de l'intensifier (par la prise d'un congé parental notamment). Les résultats sont présentés en détail après une brève synthèse de la littérature quant aux évolutions contemporaines des rôles parentaux dans les sociétés occidentales et aux conceptions générales de la paternité dans les sociétés africaines.

\section{Rattachement des auteurs \\ ${ }^{1}$ Université de Mons, Belgique}

\section{Correspondance}

dimitri.cauchie@umons.ac.be

\section{Mots clés}

paternité, périnatalité, compétence éducative, migration, Afrique centrale

\section{Pour citer cet article}

Cauchie, D., Bruyninckx, M. et Cappalonga, O. (2015). Vécu de la paternité et implication dans les soins périnataux. Rencontres avec des hommes africains émigrés en Belgique. Alterstice, 5(1), 35-44. 


\section{Introduction}

Les fonctions sociales liées à la maternité et à la paternité s'envisagent différemment dans les cultures européennes et africaines. Généralement, dans de nombreuses régions d’Afrique subsaharienne, les pères, par le partage social et culturel des rôles parentaux, ne suivent pas l'évolution de la grossesse et ne participent pas à l'accouchement : les femmes sont entourées de la famille "féminine", qui se charge de les aider au domicile pour les tâches ménagères et pour les soins du bébé. L'intrusion masculine dans ces activités est souvent socialement mal considérée. Or, en situation de migration, les femmes se retrouvent la plupart du temps seules avec leur mari, ou avec quelques membres de la famille. Le soutien des tantes, sœurs ou cousines se fait moindre et le fonctionnement familial se nucléarise. Dans un tel contexte, les hommes s'en tiennent-ils au respect des traditions culturelles d'origine ou s'impliquent-ils davantage dans la préparation à la naissance et dans les soins périnataux, ainsi que la tendance européenne le préconise?

\section{Les conceptions de la paternité : quelques repères}

\section{L'évolution socioculturelle des rôles parentaux et la paternité contemporaine en Europe occidentale francophone}

Lors de l'arrivée d'un enfant, les femmes sont généralement au centre de l'attention: elles le portent pendant neuf mois, le sentent bouger dans leur ventre, le mettent au monde... En 1980, This écrivait, montrant que les hommes se tenaient, ou restaient tenus, à distance de la grossesse et des soins périnataux, que " trop souvent encore, la naissance d'un enfant reste l'affaire exclusive d'une femme et d'un médecin; le père éliminé est traité comme un gêneur: état de fait qui n'est bon pour personne, et qu'il faut changer » (p.4). Aujourd'hui, cet "état de fait » semble avoir évolué et de nombreux hommes, notamment en Europe occidentale, assistent à l'accouchement, pratiquent l'haptonomie, revendiquent leur rôle de père. Le Camus (2000) distingue quatre registres dans lesquels les pères peuvent se situer: biologique (le géniteur), légal (I'homme qui inscrit juridiquement l'enfant dans sa lignée), social et affectif (celui qui éduque l'enfant), symbolique (celui qui fait fonction de représentant de la règle). Pour Castelain-Meunier (1997, p. 5), la paternité est donc " une construction symbolique, économique, sociale, culturelle, juridique, éthique, d'un lien. II y a toujours eu dans les différentes sociétés et à différentes périodes de I'histoire, une pluralité de pères ". En effet, chaque homme vit sa paternité différemment en fonction de sa personnalité, de sa culture, de ses croyances...

L'entrée dans la parentalité repose aujourd'hui davantage sur l'élaboration réfléchie d'un projet conjugal partagé par deux individus que sur un prescrit socioculturel. La conception même d'un enfant est devenue une affaire privée qui concerne les deux parents dans leur individualité (Castelain-Meunier, 2001). Elle n'est plus uniquement soumise aux impératifs de production d'une famille ou d'une nation, induisant la fierté d'avoir une descendance nombreuse (Frascarolo, 2001).

Les fonctions et le rôle paternels ont donc évolué, socialement et culturellement, pour être redéfinis dans une nouvelle conception de la paternité. Différents auteurs soulignent ainsi le déclin de l'autorité paternelle dans la structure familiale ainsi que celui de la fonction patriarcale (Hurstel, 2001, Le Camus, 2000). Alors qu'ils ont longtemps symbolisé l'autorité, la raison, l'exigence d'obéissance et de respect, les hommes s'inscrivent aujourd'hui dans de nouveaux modèles de paternité et décident donc des attitudes à tenir : "les conceptions des pères contemporains sont comprises entre le type de liens ayant existé avec leur propre père et les représentations contemporaines » (Castelain-Meunier, 2001, p. 29).

Si cette évolution de la paternité tient à celle des désirs des hommes quant à leur rôle parental, elle est aussi à mettre en lien avec celle du statut socioculturel des femmes et de leur place au sein de la société. En effet, l'accès croissant des femmes au marché du travail et la professionnalisation massive du travail féminin sont l'une des causes évoquées pour comprendre la redéfinition des rôles parentaux. En Belgique, en 2012, les femmes représentent $45,5 \%$ de la population active (Statbel, 2013). En conséquence, elles réorganisent leurs fonctions maternelles et les hommes se voient davantage sollicités pour la gestion domestique de la famille et les soins à apporter aux enfants : " ainsi, la professionnalisation des femmes s'accompagne d'une réorientation marquée des 
représentations collectives sur les rôles familiaux et la répartition des activités dans le couple et la famille » (Neyrand, 2000, p. 5).

Les hommes occupent donc une place croissante dans les phases périnatales et l'accouchement. Ils revendiquent actuellement davantage leur rôle de père et souhaitent être présents précocement pour leur enfant : ils sont présents lors des échographies et de l'accouchement, pratiquent l'haptonomie et procurent des soins au nouveauné. Le père n'est plus uniquement le représentant de l'autorité, il donne également de l'affection et s'occupe de son enfant. En conclusion, nous pouvons retenir que le père s'engage différemment depuis quelques décennies dans la grossesse, l'accouchement et le post-partum, par choix personnel et par adaptation aux évolutions sociétales engendrant des modifications des modèles familiaux.

\section{Les représentations traditionnelles de la paternité dans les sociétés africaines}

Les conceptions sociales et culturelles de la famille dans les sociétés africaines diffèrent de celles véhiculées au sein des sociétés occidentales. Ces dernières privilégient une construction de type «nucléaire " alors que les configurations familiales africaines sont majoritairement bien plus étendues. À cette première caractéristique, Locoh $(1988$, citée par Aryee, 1999) en ajoute d'autres, dont la stricte séparation des rôles et des responsabilités entre homme et femme, la prédominance du lignage sur la solidarité conjugale et la domination des aînés. Selon Geber, la famille n'est en outre qu'une partie d'une organisation clanique qui « comprend toutes les personnes descendant d'un même ancêtre et qui ont en commun le même symbole clanique (totem), le même chef, le même patrimoine de noms » (1998, p. 56). Le peuple Baluba, présent en République démocratique du Congo (Katanga, Kasaï, Kivu et Maniema), nomme cet ensemble " diku »: " ceux qui ont rang d'enfant se traitent entre eux de frères et de sœurs comme s'ils étaient issus d'un même père et d'une même mère " (Mfuadi, 2004, p. 24). Toutefois, beaucoup de familles africaines sont influencées à la fois par la tradition et la modernité : actuellement, en milieu urbain, la famille tend à se rétrécir et l'individualité y prime sur la solidarité, très présente autrefois (Adepoju, 1999). Cependant, l'unité familiale reste toujours la référence stable dans la plupart des populations africaines et, dès sa naissance, l'enfant est en contact avec tous les membres de la famille élargie qui participent à son éducation.

Dans beaucoup de sociétés subsahariennes, la naissance des enfants est source de grande fierté pour le couple, synonyme de réalisation personnelle pour les femmes mais aussi garante de la sécurité pour l'avenir des parents : « dans le contexte culturel africain, un enfant est à la fois considéré comme un symbole de joie et comme un appui économique » (Adepoju et Mbugua, 1999, p. 65). En général, le père est tenu à l'écart de la naissance et de la vie du bébé. II peut être éloigné du couple mère-nourrisson directement après l'accouchement, la "réclusion postnatale " de la mère lui permettant de développer une relation symbiotique avec le nouveau-né (Muke, 2003). Les hommes occuperaient donc peu de place dans les périodes périnatales. Toutefois, Dupuis avance que cette distanciation du père est davantage le fait de représentations socioculturelles occidentales que de la réalité ellemême et qu'il « est en fait extrêmement impliqué dans la vie de l'enfant, dès sa conception, et mobilisé comme tel dans les activités quotidiennes » (1991, p. 79). D’après Muke, « le géniteur africain en société traditionnelle n’a pas abandonné toutes tentatives d'intrusion durant la première enfance de ses descendants » (2003, p. 70). Quoi qu'il en soit, on peut dès lors se demander comment se positionnent et agissent ces hommes africains quand ils sont amenés à concevoir leur paternité en contexte de migration.

\section{Le vécu périnatal de pères originaires d'Afrique Centrale et immigrés en Belgique : aspects méthodologiques}

\section{Objectif de recherche et échantillon}

L'objectif de notre recherche est de tenter d'appréhender le vécu d’hommes africains, originaires de la région des Grands Lacs et immigrés en Belgique, en matière de paternité et de participation au suivi pré- et post-natal. Nous nous sommes notamment intéressés à leur participation lors des examens échographiques, leur présence lors de l'accouchement et leur contribution aux soins à apporter au nouveau-né. II s'agit aussi de comprendre comment les couples d'origine africaine s'adaptent à des conditions périnatales culturellement si différentes, en tentant de cerner quelle place l'homme souhaite prendre mais aussi quel rôle sa compagne l'autorise à jouer. 
L'échantillon sélectionné pour mener à bien cette recherche est un échantillon de type occasionnel constitué de neuf sujets. Les critères d'inclusion étaient les suivants : (1) être originaire de l'Afrique des Grands Lacs (République Démocratique du Congo, Rwanda ou Burundi); (2) avoir une conjointe également originaire d'Afrique des Grands Lacs (de manière à contrôler la variable liée à la religion puisque ces pays sont majoritairement composés de non musulmans); (3) avoir immigré à l'âge adulte (de manière à avoir été suffisamment imprégné par la culture africaine avant d'arriver en Belgique francophone); (4) avoir une conjointe enceinte au moment du premier entretien de recherche et ayant accouché au moment du second; (5) s'exprimer en français.

\section{Type de recherche et instrumentation}

Notre recherche, de type exploratoire, s'est déroulée selon un protocole bien déterminé, défini en deux phases. Nous avons rencontré les sujets une première fois vers le huitième mois de grossesse. Nous avons alors procédé à la passation d'un questionnaire anamnestique et mené un entretien semi-directif. La seconde rencontre s'est déroulée environ deux semaines après la naissance. Nous avons poursuivi l'entretien semi-directif, interrogeant les hommes sur leur vécu et le souvenir de l'accouchement, et nous leur avons administré le Questionnaire d'autoévaluation de la compétence éducative parentale (QAECEP) de Terrisse et Trudelle (1998). Nous détaillons ci-après les finalités de notre instrumentation. L'anonymat absolu a été garanti aux sujets qui ont tous consenti à participer à l'étude en connaissance de cause, assurés de la stricte confidentialité des informations qu'ils transmettraient.

\section{L'enquête anamnestique}

L'enquête anamnestique a permis de recueillir des informations signalétiques telles que l'âge, la nationalité, la date d'arrivée en Belgique et l'état civil, afin de pouvoir décrire correctement notre échantillon et de vérifier que nos critères de sélection étaient bien respectés.

\section{L'entretien semi-directif}

En suscitant la parole spontanée du sujet sur un ensemble de thèmes, fixés à l'avance et consignés dans un guide, l'entretien semi-directif, par la souplesse qu'il garantit à l'échange, permet au chercheur d'orienter la récolte des données de manière naturelle et avec considération pour les propos qui lui sont adressés. II permet de recueillir, notamment, des représentations culturelles sans trop en contraindre l'expression. Le premier entretien mené avec les pères au cours de notre recherche portait essentiellement sur deux thématiques: les traditions familiales et périnatales dans le pays d'origine et le vécu associé à la grossesse menée en Belgique. Le second s'articulait autour des souvenirs de l'accouchement (selon que les pères aient, ou non, choisi ou eu la possibilité d'assister à la naissance), de la rencontre avec l'enfant et du retour au domicile. L'anonymat ayant été strictement assuré, les entretiens ont été enregistrés afin de ne perdre aucune information et de pouvoir témoigner de la plus large disponibilité dans la communication avec ces pères.

\section{Le Questionnaire d'auto-évaluation de la compétence éducative parentale}

Le QAECEP est un instrument évaluant le sentiment de compétence du parent dans son rôle d'éducateur. II est basé sur l'échelle Parenting Sense of Competence Scale de Gibaud-Wallston (1987), qui a été traduite et validée par Terrisse et Trudelle (1988). Deux versions du test existent, l'une destinée aux mères, l'autre s'adressant aux pères. $C^{\prime}$ est donc cette dernière que nous avons utilisée. La mesure de ce sentiment de compétence se base sur deux facteurs : le "sentiment d'efficacité " évalue la perception par le sujet de son rôle éducatif parental dans sa dimension instrumentale (compétence, habiletés, connaissances...) et le " sentiment de satisfaction » est, quant à lui, associé à la dimension affective de l'exercice de la parentalité (anxiété, motivation, aisance...). Le sujet obtient un score pour chacune des deux échelles, la première mesure reposant sur sept éléments, la seconde sur neuf. Pour chacun des seize énoncés du QAECEP, le répondant exprime son degré d'accord par rapport à une échelle de type Likert à six choix de réponse. La progression de ce degré d'accord étant volontairement présentée en ordre inverse dans l'outil, le sujet obtient une note d'autant moins élevée qu'il exprime son désaccord (la première étiquette, "tout à fait d'accord ", menant à une note égale à 6 , la dernière, "tout à fait en désaccord ", menant à une note égale à 1). Les deux scores obtenus évoluent selon un continuum interprétable et présenté dans le 
tableau 1. Le score global permettant de quantifier le "sentiment de compétence parentale » du sujet s'obtient par l'addition des scores obtenus aux deux sous-échelles. Sa valeur peut donc varier entre 16 et 96 et s'interprète de manière similaire à celles de ses deux facteurs constituants. Plus le score est élevé, plus le répondant a une perception positive de sa compétence en tant que parent (Terrisse et Trudelle, 1988).

Tableau 1 Interprétation catégorisée des scores au QAECEP

\begin{tabular}{|l|c|c|c|c|c|}
\hline Sentiment... & très faible & faible & moyen & élevé & très élevé \\
\hline $\mathrm{d}^{\prime}$ efficacité (score) & {$[7-13]$} & {$[14-20]$} & {$[21-30]$} & {$[31-37]$} & {$[38-42]$} \\
\hline de satisfaction (score) & {$[9-17]$} & {$[18-26]$} & {$[27-36]$} & {$[37-45]$} & {$[46-54]$} \\
\hline $\begin{array}{l}\text { de compétence parentale } \\
\text { (score global) }\end{array}$ & {$[16-31]$} & {$[32-47]$} & {$[48-64]$} & {$[65-80]$} & {$[81-96]$} \\
\hline
\end{tabular}

L'utilisation de cet instrument dans le cadre de cette étude nous donne une indication quant à la manière dont le (futur) père envisage son implication et sa participation dans le suivi de la grossesse et des soins apportés au bébé lors du retour à la maison.

\section{Analyse des données}

L'analyse des données recueillies s'est articulée en deux temps: une première approche qualitative de type individuelle et une seconde, plus quantitative, destinée à faire émerger les éventuelles grandes tendances qui se dessinent au sein de notre échantillon.

\section{Principaux résultats de recherche}

\section{L'enquête anamnestique}

Notre échantillon est composé de neuf pères originaires d'Afrique des Grands Lacs, soit quatre d'origine congolaise (RDC), trois d'origine burundaise et deux d'origine rwandaise, ce qui respecte parfaitement nos critères d'inclusion (tableau 2). Ils ont entre 32 et 45 ans (âge moyen de 37,8 ans). Tous sont de nationalité belge et sont légalement mariés (formant avec leurs compagnes des couples endogames du point de vue de leur origine géographique), à l'exception d'un sujet congolais non marié (S6). Ce dernier n'était d'ailleurs installé en Belgique que depuis 6 ans au moment de la rencontre (le temps le plus court au sein de notre échantillon), la plupart des autres sujets y vivant depuis plus de 10 ans (de 13 à 17 ans pour six d'entre eux). La moyenne globale de durée de vie en Belgique s'élève à 12,1 ans. Pour trois sujets, l'enfant à naître au moment de la rencontre était leur aîné, les autres attendant la naissance d'un cadet (enfant de $3^{\mathrm{e}}$ au $5^{\mathrm{e}}$ rang dans la fratrie).

Tableau 2 Caractéristiques de l'échantillon

\begin{tabular}{|c|c|c|c|c|c|c|c|}
\hline \multirow{2}{*}{ Sujets } & \multirow{2}{*}{$\begin{array}{l}\text { Pays } \\
\text { d'origine }\end{array}$} & \multirow{2}{*}{$\begin{array}{l}\text { Âge } \\
\text { (ans) }\end{array}$} & \multicolumn{2}{|c|}{ Arrivée et vie en Belgique } & \multirow{2}{*}{ Nationalité } & \multirow{2}{*}{ État civil } & \multirow{2}{*}{$\begin{array}{l}\text { Rang de l'enfant } \\
\text { à naître }\end{array}$} \\
\hline & & & Âge (ans) & Durée (ans) & & & \\
\hline 1 & Congo (RDC) & 32 & 19 & 13 & Belge & Marié & $4^{e}$ \\
\hline 2 & Burundi & 40 & 23 & 17 & Belge & Marié & $3^{e}$ \\
\hline 3 & Burundi & 43 & 28 & 15 & Belge & Marié & $4^{e}$ \\
\hline 4 & Congo (RDC) & 45 & 30 & 15 & Belge & Marié & $5^{e}$ \\
\hline 5 & Burundi & 35 & 22 & 13 & Belge & Marié & $1^{\mathrm{er}}$ \\
\hline 6 & Congo (RDC) & 35 & 29 & 6 & Congolaise & Cohabitant & $1^{\mathrm{er}}$ \\
\hline 7 & Rwanda & 38 & 30 & 8 & Belge & Marié & $1^{\mathrm{er}}$ \\
\hline 8 & Rwanda & 37 & 23 & 14 & Belge & Marié & $3^{e}$ \\
\hline 9 & Congo (RDC) & 35 & 27 & 8 & Belge & Marié & $3^{e}$ \\
\hline
\end{tabular}




\section{L'entretien semi-directif prénatal}

Les traditions familiales et périnatales dans le pays d'origine

Tous les sujets étaient bien au fait des traditions familiales et périnatales dans leur pays d'origine et n'ont éprouvé aucune difficulté à en parler. Tous nous ont décrit un fonctionnement familial où le père est considéré comme un chef de famille qui ne participe ni aux tâches ménagères ni aux soins des bébés, tous ces aspects de la vie de la famille étant quasi exclusivement pris en charge par les femmes. Seul l'un d'eux (S7) précise que lorsque l'épouse est enceinte, il arrive que, dans son village d'origine, certains maris se chargent de la corvée d'eau mais que cela ne constitue cependant pas une obligation. Tous nous ont dit que, dans leur culture d'origine, le père ne s'investit pas dans le suivi de la grossesse, et n'assiste pas à la naissance en tant que telle : " c'est le rôle de la maman, des sœurs, des cousines et des tantes. Un homme n'a pas sa place dans tout ça! ", témoigne l'un des pères (S9). Même l'éducation des petits-enfants appartient à la mère et aux autres femmes de la famille.

Le vécu associé à la grossesse menée en Belgique

Six couples ont pris la décision d'avoir un enfant de manière conjointe. Dans deux autres cas, c'est le père qui semble avoir pesé sur la décision et, dans le dernier, ce serait plutôt la mère. Tous les sujets ont toutefois affirmé que leur compagne gérait seule son mode de contraception. Deux sujets semblent avoir une préférence marquée pour un enfant de sexe masculin car celui-ci représente la perpétuation de "la lignée et la sécurité pour la famille » selon eux : le fait d'avoir un " héritier [qui pourra] diriger les cadets " (S6) reste un souhait important pour ces deux hommes dont l'un (S1) souhaite également avoir un garçon car, n'ayant vécu qu'avec ses sœurs, il souhaite que sa famille en comprenne au moins un. Un seul sujet (S8) espère une fille, car il a déjà deux garçons. Pour les autres, le sexe de l'enfant à venir n'a aucune importance, notamment quand ils ont déjà des enfants des deux sexes. Quatre sujets ont choisi un prénom biblique pour leur futur bébé, deux autres ont préféré des prénoms considérés comme étant " modernes ». Les trois autres ont choisi de donner le prénom d'un aïeul (grands-parents). Tous souhaitent avoir, à terme, une grande famille. Les enfants sont en effet, pour eux, synonymes de prospérité, de bénédiction et de joie, même si le coût financier qu'ils représentent en Belgique n'est pas négligeable. Certains avancent des considérations religieuses pour justifier leur envie d'assurer leur descendance : " comme l'a demandé Dieu dans la Bible, l'union entre un homme et une femme doit se concrétiser par la venue d'un enfant ". En matière de répartition des rôles, quatre sujets estiment que "les pères et les mères ont des rôles complémentaires » et que " chacun doit donc les assumer selon ses compétences ". Cinq d'entre eux estiment que le père " dirige la famille » tandis que la femme s'occupe des enfants et du ménage. Cependant, tous déclarent aider leur compagne au quotidien car, en Belgique, la famille se nucléarise et la femme a donc moins le soutien de sa famille élargie et il est donc nécessaire de pallier cette situation : « en Afrique, la femme appartient à toute l'entité familiale dans le cadre de l'entraide; ici ce n'est pas de même! ". Tous les sujets ont l'intention de confier le bébé à venir à une infrastructure d'accueil en journée car les deux parents travaillent (à l'exception de S6, sans emploi) mais six d'entre eux accepteraient toutefois de prendre un congé parental pour s'occuper de leur enfant. Seuls quatre hommes estiment que l'éducation des filles et des garçons doit être identique. Durant la grossesse, cinq pères ont assisté aux différents examens échographiques. Parmi les quatre qui n’ont pas pu le faire, trois en ont été empêchés par des obligations professionnelles (S1, S4, S9) et un a estimé que ce moment était trop intime et que sa présence aurait pu gêner sa compagne (S2).

\section{L'entretien semi-directif postnatal}

Les souvenirs de l'accouchement et la rencontre avec l'enfant

Sept sujets sur neuf étaient présents lors de l'accouchement et, pour tous, cette présence résultait d'une volonté personnelle. Pour l'un d'eux (S4), cette naissance constituait une expérience inédite car il n'avait pas assisté à celles de ses autres enfants. Bien qu'ayant souhaité être présent, un des pères rencontrés (S3) a finalement été écarté lors de l'accouchement en raison d'une intervention réalisée en urgence (césarienne). S'il reconnaît qu'il aurait été présent principalement pour satisfaire au souhait de sa compagne, il exprime néanmoins sa frustration de n'avoir pas pu assister à la naissance de leur enfant. Enfin, le dernier sujet (S2) n'a pas souhaité être présent, préférant s'abstenir par pudeur. Tous ces pères estiment qu'accoucher en Belgique permet une plus grande sécurité sanitaire 
car la médecine y est performante et les infrastructures bien équipées. En revanche, l'absence de la famille élargie lors de la naissance engendre, selon eux, une insécurité affective certaine pour la femme mais aussi pour le père. Sept ont explicitement admis avoir pris conscience de la souffrance endurée par la femme lors de l'accouchement et avoir ressenti à leur égard un grand sentiment d'impuissance: "maintenant que j'ai vu ce qu'était un accouchement, j'ai plus de respect pour les femmes ». Être présent lors du premier cri de l'enfant, pouvoir couper le cordon ombilical, donner le premier bain, tous ces événements ont engendré un sentiment très fort chez eux et ils estiment que ces premiers contacts ont été très importants pour les liens d'attachement: « ma fille, je lui donnerai tout ce qu'un papa peut offrir ». Huit de ces hommes ont déclaré s'être sentis pères dès la grossesse de leur compagne et, pour trois d'entre eux, ce sentiment s'est progressivement développé et a surtout été exacerbé lors de la naissance de l'enfant. Tous les pères déclarent exprimer librement leurs sentiments à l'égard de leurs enfants, ne pas hésiter à les câliner et à leur parler avec douceur. Ils arrivent à se projeter dans l'avenir avec eux et s'inscrivent déjà dans un projet éducatif.

\section{Le retour au domicile}

Tous les sujets déclarent avoir aidé leur compagne lors du retour à domicile. Ils disent contribuer au bon fonctionnement du quotidien, participer aux tâches ménagères et s'occuper du bébé. Pour certains, le retour au domicile après l'euphorie de la naissance a été vécu comme quelque peu décevant en raison de l'absence de la famille élargie : " en Afrique, quand un enfant naît, c'est la fête dans tout le village; ici, chacun rentre chez soi... ". Certains ont tenté de créer cette ambiance festive à distance en envoyant des photos de leur enfant par Internet dès le jour de la naissance.

\section{Le Questionnaire d'auto-évaluation de la compétence éducative parentale}

Les résultats obtenus au QAECEP montrent que les pères interrogés éprouvent un sentiment de compétence parentale global plutôt élevé (tableau 3). Les deux facteurs sous-jacents, leurs sentiments d'efficacité et de satisfaction, étant généralement évalués dans une propension similaire. En effet, en moyenne, le score global atteint 74,2 (soit $77,31 \%$ de la note maximale, 96), ce qui correspond au quatrième niveau du sentiment de compétence (sur 5). Les pères évaluent leur sentiment d'efficacité à 32,22 (soit 76,72\% de la note maximale à la sous-échelle, 42 ) et celui de satisfaction à 42,00 (soit 77,78 \% de la note maximale à la sous-échelle, 54).

Tableau 3 Résultats au Questionnaire d'auto-évaluation de la compétence éducative parentale (QAECEP)

\begin{tabular}{|l|l|l|l|l|l|l|}
\hline \multirow{2}{*}{ Sujets } & \multicolumn{2}{l|}{ Sentiment d'efficacité } & \multicolumn{2}{l}{ Sentiment de satisfaction } & \multicolumn{2}{l|}{ Sentiment de compétence parentale } \\
\cline { 2 - 7 } & Score & Niveau & Score & Niveau & Score & Niveau \\
\hline 1 & 31 & Élevé & 27 & Moyen & 58 & Moyen \\
\hline 2 & 33 & Élevé & 45 & Élevé & 78 & Élevé \\
\hline 3 & 31 & Élevé & 34 & Moyen & 65 & Élevé \\
\hline 4 & 35 & Élevé & 46 & Très élevé & 81 & Très élevé \\
\hline 5 & 33 & Élevé & 44 & Élevé & 77 & Élevé \\
\hline 6 & 30 & Moyen & 44 & Élevé & 74 & Élevé \\
\hline 7 & 33 & Élevé & 47 & Très élevé & 80 & Élevé \\
\hline 8 & 31 & Élevé & 45 & Élevé & 76 & Élevé \\
\hline 9 & 33 & Élevé & 46 & Très élevé & 79 & Élevé \\
\hline Moyennes & 32,22 & \multirow{2}{*}{ Élevé } & 42,00 & \multirow{2}{*}{ Élevé } & 74,2 & Élevé \\
\cline { 2 - 4 } \cline { 5 - 6 } & 1,47 & 6,43 & 7,27 & & \\
\hline
\end{tabular}

À priori, ces premières tendances globales pourraient donc laisser penser que les sujets s'autoévaluent de manière assez homogène quant à ces deux dimensions de leur rôle paternel. Toutefois, comme on peut l'observer dans ce tableau, les dispersions des résultats à chaque échelle (mesurées par l'écart type des scores impliqués) sont 
sensiblement différentes: les pères se montrent en effet beaucoup moins dispersés en ce qui concerne leur sentiment d'efficacité (écart type de 1,42) que leur sentiment de satisfaction (écart type de 6,43). Par ailleurs, nous n'observons qu'une relation faible entre leurs scores respectifs à ces deux sous-échelles (le coefficient $r$ de BravaisPearson calculé sur les deux séries de données ne s'élevant qu'à 0,49). Ainsi, bien que les tendances globales que nous présentons ici émergent de manière assez claire et reflètent le vécu d'une majorité de ces pères, ces analyses préalables montrent qu'il importe de rester prudent dans l'interprétation des résultats et de ne pas généraliser abusivement ces premières observations concernant la manière qu'ont les sujets d'envisager leur rôle parental: des spécificités individuelles apparaissent et doivent être prises en compte dans l'étude des vécus subjectifs.

De manière générale, on constate que sept pères sur neuf expriment un sentiment de compétence parentale de niveau "élevé » (tableau 3). Parmi eux, trois estiment de manière équivalente, et à ce même niveau, leur sentiment d'efficacité et leur sentiment de satisfaction (S2, S5 et $\mathrm{S} 8$ ), tandis que trois se sentent davantage satisfaits qu'efficaces (avec des niveaux s'élevant, respectivement, à " très élevé " et " élevé " pour S7 et S9 et à " élevé » et " moyen » pour S6) et que la configuration inverse se présente pour le dernier (S3), qui exprime un sentiment plus important en termes d'efficacité (" élevé ») que de satisfaction (" moyen »). Les deux derniers sujets se distinguent sur le plan de leur sentiment de compétence parentale, l'un présentant un niveau inférieur à la majorité de l'échantillon (S1: " moyen », en se sentant plus efficace que satisfait), l'autre présentant un niveau supérieur (S4 : "très élevé ", en se sentant, lui, plus satisfait qu'efficace). Si la plupart des sujets s'estiment donc plutôt compétents dans leur rôle paternel, le détail de nos résultats montre cependant que, quand ils se positionnent à des niveaux différents quant à leurs sentiments d'efficacité et de satisfaction, c'est ce dernier qui prédomine dans quatre cas sur six. De même, une analyse plus approfondie des scores aux sous-échelles (relativement à leurs notes maximales respectives) montre que cette même configuration caractérise les trois pères présentant des résultats équivalents pour les deux facteurs (quand ils sont catégorisés). Pour sept des neuf pères rencontrés, c'est donc principalement la dimension affective de l'exercice de la paternité, leur aisance dans ce rôle, qui « nourrit » leur sentiment de compétence et y contribue, plus que la dimension instrumentale de leur fonction parentale, et ce, bien que nous ayons montré que le degré de perception des sujets quant aux habiletés et aux connaissances qu'ils ont acquises pour être un parent adéquat ne s'avérait pas faible pour autant. Notons encore que les résultats au QAECEP semblent dépendants de l'expérience préalable de la paternité pour les sujets. En effet, le seul père évaluant son sentiment de compétence parentale comme étant " très élevé » est celui dont l'enfant né durant notre recherche était le cinquième de la famille (S4). Et en ce qui concerne les deux sujets de notre échantillon faisant prédominer leur sentiment d'efficacité, on peut observer qu'il s'agit des deux qui sont devenus pères pour la quatrième fois. Enfin, la durée de vie passée en Belgique pourrait également ne pas être sans influence sur une part constituante du sentiment de compétence de ces pères immigrés : on peut ainsi relever que le sujet s'estimant le moins efficace dans son rôle paternel (S4) est celui qui vit en Belgique depuis seulement 6 ans (soit le plus récemment immigré) et dont l'enfant est premier né. Globalement, les résultats au QAECEP sont cohérents avec les discours des pères, qui se disent à l'aise avec leur enfant et participent volontiers aux soins à apporter. Ils n'ont pas peur de le manipuler et apprécient le contact physique avec lui. Ils se sentent investis dans leur rôle paternel et engagés dans le projet éducatif qui s'amorce avec cette naissance. Ces hommes sont plutôt fiers de ce qu'ils sont en tant que pères.

\section{Discussion des résultats et conclusions}

Différents facteurs associés à la "modernisation" (occidentalisme, colonisation, migration des hommes, urbanisation, etc.) ont quelque peu fait évoluer les conceptions culturelles quant aux rôles sociaux qu'occupent I'homme et la femme dans diverses sociétés africaines. La différenciation de genre des fonctions parentales reste cependant assez fortement marquée. Avec cette étude, nous avons cherché à mieux comprendre comment, dans une situation interculturelle, des pères africains originaires d'Afrique des Grands Lacs et immigrés en Belgique concevaient et vivaient leur paternité.

Nos résultats montrent que ces pères restent bien conscients du statut social lié à la paternité dans leurs conceptions d'origine (autorité, "auto-exclusion" relative de l'éducation des enfants, etc.) mais font preuve d'adaptation culturelle dans l'exercice de ce rôle dans le contexte sociétal d'accueil. Ainsi, ils remplissent des fonctions traditionnellement dévolues aux femmes. Leurs compagnes étant professionnellement actives, ils 
estiment d'ailleurs normal de contribuer à la gestion domestique du ménage. Toutefois, nous avons également montré que ces hommes ne partagent pas tous les mêmes opinions quant à une distinction genrée des rôles éducatifs parentaux, chacun disposant de compétences spécifiques. Parmi ceux témoignant de changements comportementaux, certains ne les maintiendraient d'ailleurs probablement pas dans leur contexte originel. Ils pensent que ce changement culturel dans la répartition des rôles est notamment rendu possible par le fait que les membres de leur famille étendue vivent toujours en Afrique Centrale et ils ne peuvent dès lors pas porter de jugement sur leur manière d'agir.

Malgré l'impact financier important d'une naissance dans une famille, les sujets restent, dans la plupart des cas, attachés au désir d'avoir une famille nombreuse. Tous les pères dénonçant l'insécurité affective que représente l'absence de la famille élargie dans le déroulement des grossesses, nous pouvons peut-être voir dans ce désir le souhait de perpétuer une tradition culturelle, la volonté de reconstituer un réseau familial plus vaste. Par ailleurs, la sécurité médicale et sanitaire dont peut bénéficier une (future) mère au sein de la société d’accueil est probablement un facteur ne réfrénant pas ce désir d'enfants.

Enfin, en ce qui concerne le sentiment de compétence parentale des sujets rencontrés, nous avons pu montrer qu'il était relativement élevé, la " satisfaction affective " primant d'ailleurs légèrement sur l' " efficacité instrumentale ». Pour Gomes-da-Costa et coll. (2011), la mise en œuvre par un individu de sa compétence parentale serait "fortement conditionnée par les contextes dans laquelle elle s'exerce » (p. 182) et notamment par des facteurs sociodémographiques et socioculturels. On peut dès lors s'interroger à propos de l'effet éventuel sur ces sentiments de compétence de l'intégration de ces migrants dans une société d'accueil dans laquelle les conceptions sociales et culturelles de la parentalité diffèrent parfois largement de leurs représentations originelles.

Les hommes rencontrés se sont, dans la plupart des cas, installés seuls en Belgique. Ils ont donc été amenés à se débrouiller par eux-mêmes. La transition des traditions d'origine vers celles de leur nouvel environnement de vie était donc déjà entamée avant leur mariage et la naissance des enfants. Aujourd'hui, quasiment tous possèdent la nationalité belge et rares sont ceux qui envisagent un retour en Afrique. Ils ressentent dès lors la nécessité de se resituer par rapport aux questions familiales et de parentalité qu'ils se posent. Les hommes rencontrés lors de cette étude semblent parvenir à trouver des repères dans leur nouvel environnement de vie et à élaborer une série de rôles inédits, et ce, sans trop de difficultés.

Pour conclure, rappelons que le désir d'enfant chez l'homme et sa conception de la paternité se manifestent différemment selon l'expérience de chacun : " vouloir cerner le désir d'enfant chez le père (comme chez la mère d'ailleurs) relève de la mythologie car les motivations de chacun des membres du couple plongent dans des racines multiples, conscientes et inconscientes dans le déroulement de leur propre vie passée et en devenir » (ColonnaCésari, 1990, p. 17). Pour les hommes rencontrés, la redéfinition de leur rôle paternel peut sembler tributaire de l'évolution du statut des femmes. Ces dernières étant davantage intégrées sur le plan socioprofessionnel, la réorganisation des fonctions parentales amènerait les pères à s'impliquer davantage dans la préparation à la naissance et dans l'éducation des enfants. Y sont-ils, consciemment ou non, "contraints " par les nouvelles modalités de fonctionnement conjugal auxquelles ils doivent s'ajuster? Ou la configuration culturelle de la société d'accueil leur offre-t-elle l'opportunité de concrétiser un désir paternel personnel qui, peut-être, ne pouvait se manifester de manière équivalente dans leur contexte d'origine? Les résultats de notre recherche laissent penser que si la première hypothèse expliquerait " pragmatiquement » une réorganisation des fonctions parentales, la seconde n'est pas à écarter, notamment eu égard à la satisfaction exprimée par ces hommes quant à l'exercice de leur rôle de père dans des circonstances relativement nouvelles ou la perspective positive d'un congé parental, traduisant selon nous une volonté intrinsèque d'implication personnelle davantage proactive dans les soins périnataux de leur enfant. 


\section{Références bibliographiques}

Adepoju, A. (1999). La famille africaine. Politiques démographiques et développement. Paris : Karthala.

Adepoju, A. et Mbugua, W. (1999). Les mutations de la famille africaine. Dans A. Adepoju (dir.), La famille africaine. Politiques démographiques et développement (p. 59-84). Paris : Karthala.

Aryee A. F. (1999). L'évolution des modèles matrimoniaux. Dans A. Adepoju (dir.), La famille africaine. Politiques démographiques et développement (p. 109-134). Paris: Karthala.

Castelain-Meunier, C. (1997). La paternité. Paris : Presses universitaires de France.

Castelain-Meunier, C. (2001). La nouvelle présence du père. Revue des deux mondes, 5, 28-34.

Colonna-Césari, C. (1990). La grossesse du père. Paris : Chiron.

Dupuis, A. (1991). L'homme musombi : la couvade chez les Nzebi (Gabon). Dans S. Lallemand, O. Jouret, E. Ewombe-Moundo, B. Ravololomanga, A. Dupuis, M. Cros et D. Jonckers (dir.), Grossesse et petite enfance en Afrique noire et à Madagascar (p. 77-101). Paris : L'Harmattan.

Frascarolo, F. (2001). Ancrages pour la paternité. Dans C. Zaouche-Gaudron (dir.), La problématique paternelle (p. 163-176). Ramonville-Saint-Agne : Éditions Érès.

Gomes-da-Costa, J., Antunes, C., Além, A., Gouveia, L., Castro, E. et Azevedo, C. (2011). Questionnaire d'autoévaluation de la compétence éducative parentale (Terrisse et Trudelle, 1988) : application sur un échantillon de parents d'adolescents portugais. Dans E. Catarsi et J.-P. Pourtois (dir.), Éducation familiale et services pour l'enfance (p. 180-186). Florence : Firenze University Press.

Geber, M. (1998). L'enfant africain dans un monde de changement. Étude ethno-psychologique dans huit pays sudafricains. Paris : Presses universitaires de France.

Hurstel, F. (2001), Les fonctions du père dans la société contemporaine postindustrielle : enjeux anthropologiques et subjectifs. Dans C. Zaouche-Gaudron (dir.), La problématique paternelle (p. 61-74). Ramonville-SaintAgne : Éditions Érès.

Le Camus, J. (2000). Le vrai rôle du père. Paris : Odile Jacob.

Mfuadi, T. (2004). Coutumes et traditions baluba. Paris : L'Harmattan.

Muke, S. (2003). La place du père africain dans la période périnatale. Dans P. Marciano (dir.), Le père, I’homme, le masculin en périnatalité (p. 65-72), Ramonville-Saint-Agne : Éditions Érès.

Neyrand, G. (2000). L'enfant, la mère et la question du père. Paris : Presses universitaires de France.

Statbel (2013). Le marché du travail en Belgique en 2012. Bruxelles : SPF Économie, PME, classes moyennes et énergie, Direction générale Statistique et Information économique.

Terrisse, B. et Trudelle, D. (1988). Le Questionnaire d'auto-évaluation de la compétence éducative parentale (QAECEP). Montréal : Groupe de Recherche en Adaptation scolaire et sociale, Université du Québec à Montréal.

This, B. (1980). Le père : acte de naissance. Paris : Seuil. 